\title{
Hot Spots Detection of Operating PV Arrays through IR Thermal Image Using Method Based on Curve Fitting of Gray Histogram
}

\author{
Jiang $\operatorname{Lin}^{1, a}$, Su Jianhui ${ }^{1}$ and Li Xin ${ }^{1}$ \\ ${ }^{1}$ Electrical Engineering and Automation School, Hefei University of Technology, Hefei, Anhui, China
}

\begin{abstract}
The overall efficiency of PV arrays is affected by hot spots which should be detected and diagnosed by applying responsible monitoring techniques. The method using the IR thermal image to detect hot spots has been studied as a direct, noncontact, nondestructive technique. However, IR thermal images suffer from relatively high stochastic noise and non-uniformity clutter, so the conventional methods of image processing are not effective. The paper proposes a method to detect hotspots based on curve fitting of gray histogram. The result of MATLAB simulation proves the method proposed in the paper is effective to detect the hot spots suppressing the noise generated during the process of image acquisition.
\end{abstract}

\section{Introduction}

Hot spots of PV modules can be caused by poor cell matching, cracks, localized soiling (bird droppings), or shadowing ${ }^{[1]}$. A hot spot can occur when a cell (or cells) are forced into reverse bias because it (they) cannot carry the peak power current being produced by the other cells in series. Cells are supposed to be protected by the bypass diodes while the bypass diodes often fail or are never installed correctly. The performance and lifetime of PV system are affected by hot spots seriously ${ }^{[2]}$.

Hot spots make the surface temperature of $\mathrm{PV}$ module rise abnormally so that they can be detected by infrared thermography. The detection of hot spots in PV modules through thermal image is a direct, noncontact, nondestructive technique which can not only get more accurate results, but also locate the fault point to evaluate the efficiency.

J.A. Tsanakas, etc. obtain thermal images of an operating photovoltaic array by field infrared thermographic measurements. The thermal images are analyzed by means of temperature line profiles, image histogram features and the results reveal the presence of several hot spots in the four modules of the array that are all associated with specific discontinuous cells ${ }^{[3]}$. Then, J.A. Tsanakas, etc. proposes the use of standard thermal image processing and the Canny edge detection operator as diagnostic tools for module-related faults that lead to hot-spot heating effect ${ }^{[4]}$. Wang Pei-zhen presents a new method of target feature extracting in view of low contrast of target and background, strong noise and non uniformity in the infrared image and peculiarity of the photovoltaic array. First, the subtraction operation is carried out between the two infrared images of photovoltaic array which are to be analyzed and under normal condition respectively. Then, a method of combining the two dimensional threshold and the fuzzy means algorithm is implemented ${ }^{[5][6]}$.

However, there are still several key problems needed more experiments to research such as the interference of complex environment and noise. It has been found in the experiments that due to some factors such as reflecting sunlight, the thermal image of the PV arrays will appear false hot spots to cause "false alarms"; on the other hand, the emissivity of the glass on PV modules varies with the shooting angel to cause the thermal radiation quantity received by the thermal imager changed that may be conflicting with the actual variations related to hot spots and, thus, cause "false alarms"[7]. So it is necessary to suppress noise in the thermal image to accurate hot spots detection.

If the quantity of thermal image is good enough with little noise, the hot spots can be detected successfully using the method proposed by J.A. Tsanakas and Wang Peizhen etc. However, even If the images are preprocessed with Gauss filter or media filter, the hot spots can't be detected successfully from a thermal image with a large amount of noise.

In this paper, the method based on curve fitting of gray histogram is proposed in thermal image processing to suppress noise and detect hot spots accurately. Experiments and research on the interference of the IR thermal image of the PV module (noise sources in complex environment and image acquisition) are carried out.

\section{Method based on curve fitting of gray histogram to detect hot spots}

\footnotetext{
$\overline{{ }^{a} \text { Corresponding author: jianglin_hfut } @ 126 . c o m ~}$
} 
The gray level histogram of image is a function of the gray level, which is described as the number of pixels with a gray level in the image: its horizontal coordinate is gray level, and the vertical coordinate is the frequency of the gray scale (the number of pixels in the digital image). "Peak"on histogram shows the frequency of the gray level is relative large, whereas "valley" is illustrated in that the probability of the gray level appears small.

Fig. 1 is an infrared thermal image of photovoltaic module. Fig. 2 is the gray histogram of Fig.1. It can be seen from the two figures that the backgrounds, the normal working part and the hot spots of the PV module have obviously different gray levels which correspond to multiple "peaks" and "valleys" in the gray histogram obviously. As marked in the figure, the normal working part of the PV module corresponds to the highest "peak" of the gray histogram, and the hot spots correspond to lower "peak" of the gray histogram.

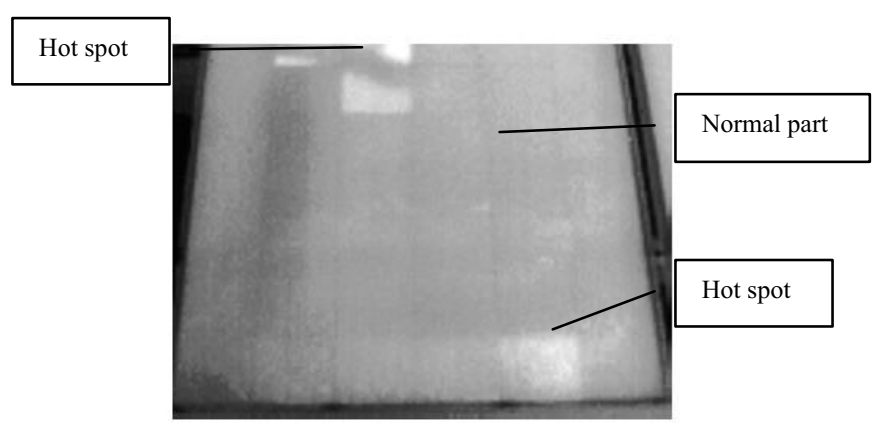

Figure 1. Infrared thermal image of PV module

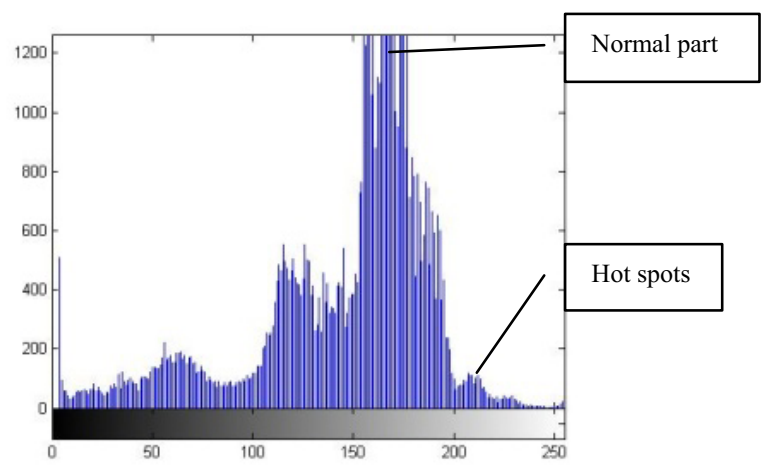

Figure 2. Gray histogram of Fig.1

Due to the influence of noise and environment interference to the infrared image, the gray histogram exists burrs and small local ups and downs, so in many cases it is difficult to detect the interest "peak" by conventional methods robustly and accurately. In order to solve this problem, this paper proposes a new image segmentation method based on curve fitting of gray histogram which is used to detect hot spots of PV modules rapidly and accurately. The method is described as follows:

Firstly, the infrared thermal image of the PV module is preprocessed. Because the thermal image is easy to be polluted by noise, the Gauss filter is used to filter the image. Gauss filter is a kind of linear smoothing filter based on the shape of Gauss's function which is effective to suppress the noise subjected to the normal distribution.

Secondly, the gray histogram of the infrared image is identified and determined. The vertical coordinate system for the histogram is set: the positive direction of the $\mathrm{X}$ axis is set to the increase of the gray value, the $\mathrm{Y}$ axis is marked by the frequency of gray level. So the gray histogram of the infrared image is established as formula (1):

$$
\mathrm{y}=p(x) \quad x=0,2,3 \mathrm{~K} 255
$$

\section{$x$ : gray levels of 8 bit gray scale digital image}

$$
y \text { : the number of pixels of a gray level }
$$

Due to the influence of noise and environment interference to the infrared image, the gray histogram exists burrs and small local ups and downs, so it is difficult to detect the interest "peak" and "valley" by conventional methods. Therefore, the least square method is used to fit the gray level histogram. The gray level histogram provides us with 256 sample points whose coordinates are seen as formula (2) :

$$
\left(x_{i}, y_{i}\right), i=0 \mathrm{~K} 255, x_{i}=i, y_{i}=p\left(x_{i}\right)
$$

According to the principle of least square method, a curve $y=\phi(x)$ is found which makes the sum of squares of deviation [formula (3)] between the curve and the sample points minimal. The histogram is fitted as a smooth curve eliminating the burrs and local fluctuations. The fitting curve of gray histogram of Fig. 2 is shown in Fig.3.

$$
\sum_{\mathrm{i}=1}^{256} \delta_{\mathrm{i}}^{2}=\sum_{\mathrm{i}=1}^{256}\left(\phi\left(x_{i}\right)-y_{i}\right)^{2}
$$

The result of first-order derivative and second derivative of the fitting curve function $y=\phi(x)$ can be used to determine the abscissa of the "peaks" and "valleys" in the histogram.

If $\phi^{\prime}\left(x_{p_{i}}\right)=0, \phi^{\prime \prime}\left(x_{p_{i}}\right)>0,\left[x_{p_{i}}, \phi\left(x_{p_{i}}\right)\right]$ is the position of "peak";

Else if $\phi^{\prime}\left(x_{v_{j}}\right)=0, \phi^{\prime \prime}\left(x_{v_{j}}\right)<0,\left[x_{v j}, \phi\left(x_{v_{j}}\right)\right]$ is the position of the "valley". 


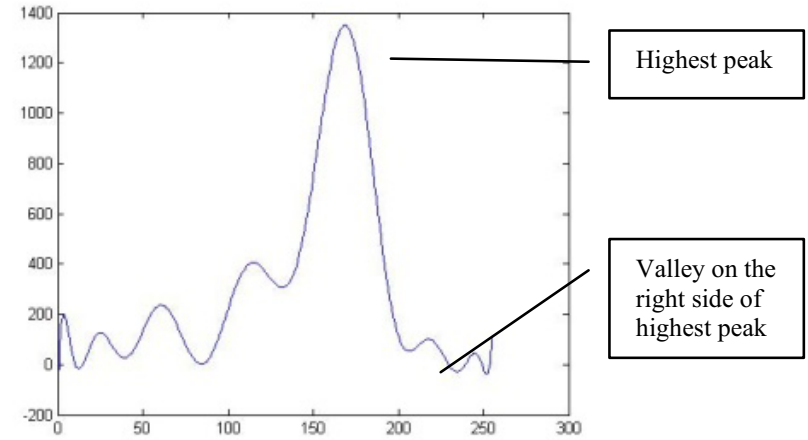

Figure 3. Fitting curve of gray histogram

Based on the characteristics of infrared thermal image of PV module, we can select gray value corresponding to the first 'valley' on the right hand side of the highest peak as the segmentation threshold, so that the hot spots can extracted.The threshold $\mathrm{T}$ is set according to formula(4).

$$
T=\min \left\{x_{v_{j}}>x_{p_{m}}\right\}
$$

$x_{p_{m}}$ is the abscissa of the highest "peaks"

$x_{v j}$ is the abscissa of all the "valleys"

\section{Experimental result}

\subsection{Hardware and software}

The experimental part of this study includes one portable infrared thermal imager regarding PV arrays installed on the rooftop of a building in Sungrow Power Supply Co., Ltd. located in Hefei, China. PV arrays consist of crystalline silicon modules, type JA SOLAR JAP6-60$255 / 3 \mathrm{BB}$. The infrared thermal camera employed for the performed measurements is SAT-S80 .

Basic processing of the obtained thermal images is performed with the use of SatIr Report software. Further processing is implemented in Matlab R2014a environment.

\subsection{Experimental outline}

The thermographic measurements took place in the city of Hefei (north-eastern China, longitude: N31 ${ }^{\circ} 52^{\prime}$, latitude: $\mathrm{E} 117^{\circ} 17^{\prime}$, mean elevation: $\left.19.05 \mathrm{~m}\right)$ at 11:30 in Nov.26th. under clear-sky conditions. The environmental conditions are given in Table 1.The inspected PV arrays are installed at a fixed angle $\theta \approx 40$
Table 1. Environmental conditions in experiment

\begin{tabular}{|c|c|c|c|}
\hline Time & temperature & illuminance & $\begin{array}{c}\text { Wind } \\
\text { speed }\end{array}$ \\
\hline $\begin{array}{c}2015 / 11 / 26 \\
11: 30\end{array}$ & $7^{\circ} \mathrm{C}$ & $45301 \mathrm{ux}$ & $3.4 \mathrm{~m} / \mathrm{s}$ \\
\hline
\end{tabular}

\subsection{Result and discussion}

In the experiment on 26 November, we collected one image of the PV module with hot spots, as shown Fig 1. It is very clear from the thermal image that some hot spots appear on the PV module which may be caused by dust or internal defects.

In the past research, the hot spots are detected by the thermal image of the PV modules, using the edge detection method based on Canny operator or threshold segmentation method or fuzzy clustering method which are effective for the thermal image in absence of noise (noise sources in the complex environment such as light, shadow or the acquisition process of thermal images). If the thermal image is good enough with little noise, the hotspots can be detected successfully using the OSTU method or Canny operator.

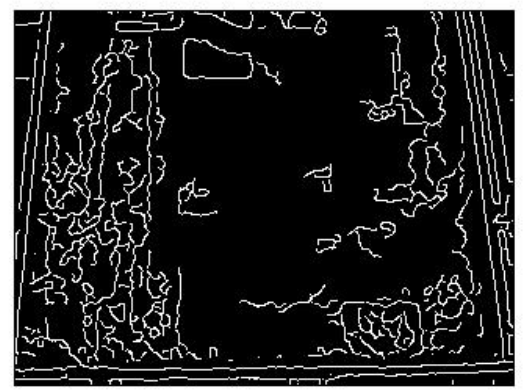

Figure 4. Canny edge detection of hot spots

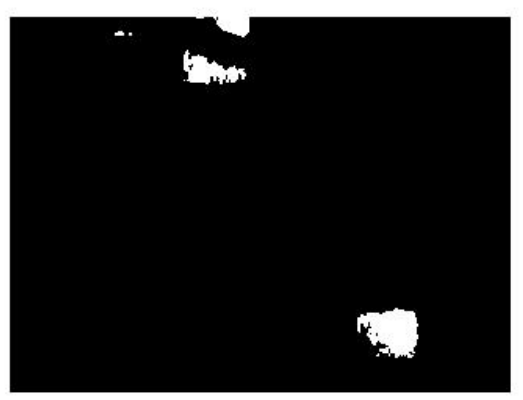

Figure 5. Hot spots detection using method of fitting curve of histogram

However, the noise in the thermal image as shown in Fig. 1 is obvious: the dark areas in Fig.1 don't always mean the low temperature, instead, the emissivity of the PV modules varying with the shooting angel causes the 
thermal radiation quantity received by the thermal imager changed; Some dark areas in Fig. 1 are the gaps between PV modules, namely ambient temperature. The noises in Fig. 1 is so obvious that even If the images are preprocessed with conventional methods such as Gauss filter or media filter, the actual effect is poor.

Fig. 1 is processed using the canny operator to result in fig. 4 in which the hot spots are detected but submerged in large of noises.

The research about the infrared thermal image with noises in the complex environment is less, so the main work in the paper is to propose a method to detect hotspots based on curve fitting of gray histogram for this kind of thermal image.

Gray histogram is a function of gray level, which means the number of pixels of each gray level in the image, that is, the frequency of each gray level appears in the image.

Fig. 2 is the gray histogram of Fig.1. Fig. 3 is the fitting curve of Fig. 2 using the fitting technique based on the least square.

It can be seen from the histogram as shown in Fig.2 that the pixels with the highest frequency of gray level mean the normal area of PV modules. So it is realized the first valley bottom of the fitting curve after its peak is the key point which can separate hot spots from normal areas of PV modules and the abscissa of the valley bottom is the threshold for segmentation.

According to the formula (4), the segmentation threshold is determined and the hot spots are extracted successfully from the thermal image as shown in Fig.5.

The result of MATLAB simulation proves the method proposed in the paper is effective to detect the hot spots suppressing the noise generated during the process of image acquisition .

\section{Conclusions}

The detection of hot spots in operating PV array through IR thermal images is studied in the paper. The experiment is made on the rooftop of a building in Sungrow Power Supply Co., Ltd. in Hefei and multi thermal images of the PV modules with hotspots are collected. It is found that the thermal images suffer from relatively high stochastic noise and non-uniformity clutter caused by the complex environment and emissivity uncertainty. From the MATLAB simulation, we can see the hotspots can't be detected using the conventional image processing method. So a new method based on curve fitting of gray histogram is proposed in this study. The result of MATLAB simulation proves the new method is effective to detect the hot spots suppressing the noise generated during the process of image acquisition.

The new method proposed in this paper need to be improved. In curve fitting of gray histogram spine function may be chose to accurate the threshold. The result of segmentation of thermal images can be classified to identify the fault of PV arrays. These are further work of our research team.

\section{References}

1. John H. Wohlgemuth, Sarah R. Kurtz, "How Can We Make PV Modules Safer? " , 2012 IEEE Photovoltaic Specialists Conference(2012)

2. Ahuja, D., and M. Tatsutani. "Sustainable Energy for Developing Countries",A report to the Academy of Sciencesfor the DevelopingWorld (TWAS) (2008)

3. J. A. Tsanakas, P. Botsaris, "On the Detection of Hot Spots in Operating Photovoltaic Arrays through Thermal Image Analysis and a Simulation Model", Materials Evaluation (2013)

4. J.A. Tsanakas, D. Chrysostomou, P.N. Botsaris and A. Gasteratos, "Fault diagnosis of photovoltaic modules through image processing and Canny edge detection on field thermographic measurements", International Journal of Sustainable Energy, 34(2015)

5. Wang Peizhen ,Zheng shicheng, "Fault Diagnosis of Photovoltatic Array Based on infrared image", Acta Energias Sinica, 31(2010) (in Chinese)

6. WANG Peizhen, WANG Qunjing, YANG Weihan, "A method for the feature extraction of the infrared image of the photovoltaic array", Journal of Hefei UniversityN of Techolygy(2004)(in Chinese)

7. Zhiyuan Zou, Yihua Hu, Bin Gao, W. L. Woo, Xinyu Zhao, "Temperature recovery from degenerated infrared image based on the principle for temperature measurement using infrared sensor", Journal of Applied Physics (2014)(in Chinese) 